\title{
Antimicrobial activity of silicone rubber used in hydrocephalus shunts, after impregnation with antimicrobial substances
}

\author{
R BAYSTON, RDG MILNER \\ From the Department of Paediatrics, Children's Hospital, Sheffield
}

SUMMARY Colonisation of cerebrospinal fluid shunts by coagulase-negative staphylococci (Staphylococcus albus) is a serious problem. Because of its possible role in prevention of the condition, the antimicrobial activity of silicone rubber after impregnation with antimicrobial drugs was studied. The method of impregnation used and test methods were found to be important. Formaldehyde-urea condensates gave no activity. Gentamicin sulphate gave activity which was short-lived. Sodium and diethanolamine fusidates and clindamycin hydrochloride gave prolonged activity. A method of impregnation was developed which could be applied to commercially available shunts before use.

Obstruction by tissue or thrombus and colonisation by micro-organisms are the two major causes of failure of implanted shunting devices used to treat hydrocephalus. The organisms causing colonisation are derived from the patient's skin at operation ${ }^{1}$ and are usually coagulase-negative staphylococci (Staphylococcus albus). The incidence of shunt colonisation varies from $3.5 \%$ to $22 \%{ }^{23}$ and its eradication almost always involves removal of the shunt. ${ }^{4}$ Previous attempts to prevent shunt colonisation, including the preoperative use of prophylactic antibiotics, ${ }^{5}$ have been unsuccessful although the use of peroperative intraventricular gentamicin is encouraging. 6 ?

These problems led us to study the effects of introducing antimicrobial substances into the silicone rubber from which the shunt components are made. Preliminary results, using a room-temperature crosslinking rubber, showed that antibacterial activity could be conferred on the rubber by mixing antimicrobial drugs with the rubber before crosslinking. ${ }^{8}$ As the silicone rubber used for shunt components is a heat-crosslinking type, further experiments were carried out to develop methods of introducing antimicrobial substances into this type of silicone rubber and to assess the antibacterial properties after the rubber was crosslinked.

\section{Material and methods}

Heat-crosslinking silicone rubber (MDX 4-4515,

Accepted for publication 11 March 1981
Dow Corning) was used throughout this work. The antimicrobial substances chosen for investigation were: gentamicin sulphate (Genticin, Nicholas Laboratories), clindamycin hydrochloride (Dalacin C, Upjohn Ltd) diethanolamine fusidate and sodium fusidate (Fucidin, Leo Laboratories), and two formaldehyde-urea condensates (Anaflex and Taurolin, Geistlich Sons Ltd). Raw heat-crosslinking silicone rubber is supplied as a stiff white putty which has to be softened by passing several times through a rubber mill (RAPRA mill, Rubber and Plastics Research Association, Shawbury) before moulding. Aliquots of milled rubber were moulded in a stainless steel mould using a 40 ton hot press (G Moore, Birmingham) at a temperature of $150^{\circ} \mathrm{C}$ for $20 \mathrm{~min}$, to produce discs of crosslinked rubber having a diameter of $70 \mathrm{~mm}$ and a thickness of $1 \mathrm{~mm}$. After the discs were removed from the mould they were heated in a circulating air oven at a temperature of $110^{\circ} \mathrm{C}$ for one hour in order to remove volatile by-products of crosslinking. ${ }^{9}$

Four methods of introducing the antimicrobial substances into the rubber were used. Method 1 consisted of mixing the drug powder into the raw rubber during milling, before it was moulded, in a concentration of $0.02 \mathrm{~g}$ to $0.98 \mathrm{~g}$ of rubber $(2 \%)$. Method 2 consisted of emulsifying a solution of rubber with a solution of the drug. $10 \mathrm{~g}$ of rubber, milled and cut into thin strips in order to aid dissolution, was added to $100 \mathrm{ml}$ of a mixture of one part of chloroform and two parts of toluene in a homogeniser (MSE) and blended at half speed in a fume cupboard. A solution of $0.32 \mathrm{~g}$ of gentamicin 
sulphate was made in $1 \mathrm{ml}$ of distilled water. $250 \mu \mathrm{l}$ of this solution was added to $40 \mathrm{ml}$ of the rubber solution and the mixture was blended at top speed for $15 \mathrm{~min}$, after which it was transferred immediately to a square $12^{\prime \prime} \times 12^{\prime \prime}$ glass assay plate and a current of air directed over the surface for one hour. The organic solvents evaporated to leave a thin sheet of raw rubber which was scraped off the plate and milled prior to moulding. The drug concentration was calculated to be $0.08 \mathrm{~g}$ in $4 \mathrm{~g}$ of rubber $(2 \%)$. Method 3 was identical to method 2 except that the $250 \mu \mathrm{l}$ of the gentamicin sulphate solution was added to $2.5 \mathrm{ml}$ of distilled water and the resulting solution was blended into the rubber solution. In method 4, raw rubber was milled and moulded as in method 1 but no drugs were added until after the crosslinking by-products had been driven off. The rubber discs were then immersed in a solution of $0.2 \mathrm{~g}$ of drug in $10 \mathrm{ml}$ of chloroform ( $2 \%$ ) for one hour, after which they were placed in a circulating air oven at $37^{\circ} \mathrm{C}$ for one hour. They were then washed twice in phosphatebuffered saline (PBS) pH 7•4, and dried.

All the antimicrobial substances except clindamycin hydrochloride were used in method 1 , as this drug was found to inhibit crosslinking, and could only be used in method 4 . Only gentamicin sulphate was used in methods 2 and 3 because the remaining suqstances were not sufficiently soluble in distilled water. Clindamycin hydrochloride and diethanolamine fusidate were used in method 4 , the remaining drugs being insufficiently soluble in chloroform.

Tests for antibacterial activity were carried out on discs $5 \mathrm{~mm}$ in diameter which had been cut from the $70 \mathrm{~mm}$ discs using a cork borer. Three discs were tested from each preparation. The discs were all rinsed twice in PBS before testing to remove any surface accumulation of drug, and one face of each disc was then marked with an indelible marker. The discs were placed marked surface uppermost upon the surface of an agar plate (Diagnostic sensitivity test (DST) Oxoid Ltd) which had previously been seeded with an overnight broth culture of Staph albus, and incubated at $37^{\circ} \mathrm{C}$ overnight. Antibacterial activity was expressed as the diameter of the zone of inhibition minus the diameter of the test disc. Where the zone diameter did not exceed that of the disc by more than $1 \mathrm{~mm}$, antibacterial activity was considered to be absent.

Tests for persisting antibacterial activity were carried out using two methods. In the first method the discs were transferred daily to fresh DST agar plates, taking care to keep the marked surface uppermost, until no more activity could be demonstrated. In the second method, discs were marked as above and immersed in $2 \mathrm{ml}$ of PBS pH 7.4 in sealed tubes and incubated on a tissue culture roller drum at $37^{\circ} \mathrm{C}$ for $18 \mathrm{~h}$. Each preparation of drugimpregnated rubber was tested in triplicate. After incubation the discs were removed from the tubes, blotted dry, and planted upon the surface of a seeded DST agar plate as in the previous method. The marked surface was placed uppermost. After the inhibition zone diameters had been recorded, the discs were removed from the plates, immersed in fresh PBS and again incubated on a roller drum. The process was repeated until no more antibacterial activity could be detected on the plates.

When the discs which had been eluted in PBS on the roller drum ceased to show activity, they were washed well in several changes of PBS and blotted dry. They were then each planted on to a separate, uninoculated DST agar plate, and overlaid with $10 \mathrm{mk}$ of cooled DST agar to which Staph albus had been? added to give a viable count of approximately $10^{6}$ colony forming units per $\mathrm{ml}$ as determined by the Miles-Mizra method. One plate from each trio was incubated immediately, and the other two were refrigerated for three and seven days respectively before incubating. After incubation, the zones of inhibition in the upper layer were measured.

\section{Results}

The results of tests for presence of antibacterial activity of silicone rubber containing drugs, and of two methods of testing for its persistence, are shown in Table 1.

Rubber containing the formaldehyde-urea conden-

Table 1 Results of testing discs of impregnated silicone rubber for antibacterial activity and its persistence

\begin{tabular}{|c|c|c|c|c|}
\hline Drug & $\begin{array}{l}\text { Method of } \\
\text { introduction }\end{array}$ & $\begin{array}{l}\text { Antibacterial activity } \\
\text { (Mean zone diameter }(\mathrm{mm}))\end{array}$ & $\begin{array}{l}\text { Persistence (days) } \\
\text { Serial plate transfer }\end{array}$ & $\begin{array}{l}\text { Persistence (days) } \\
\text { Elution in PBS }\end{array}$ \\
\hline Gentamicin sulphate & $\begin{array}{l}1 \\
2 \\
3\end{array}$ & $\begin{array}{l}16 \cdot 3 \\
9 \cdot 03 \\
0\end{array}$ & $\begin{array}{c}9 \\
4 \\
\text { ND }\end{array}$ & $\begin{array}{c}5 \\
0 \\
\text { ND }\end{array}$ \\
\hline Sodium fusidate & 1 & $23 \cdot 8$ & 38 & 13 \\
\hline Diethanolamine fusidate & 1 & $24 \cdot 2$ & 49 & 7 \\
\hline Diethanolamine fusidate & 4 & $23 \cdot 0$ & 42 & 5 \\
\hline Clindamycin hydrochloride & 4 & $18 \cdot 3$ & 13 & 9 \\
\hline Anaflex & 1 & $\mathbf{0}$ & ND & ND \\
\hline Taurolin & 1 & 0 & ND & ND \\
\hline
\end{tabular}

ND = not done 
sates showed no antibacterial activity. Rubber containing gentamicin sulphate gave results which differed according to the method of introduction of the drug. Method 1 gave larger zones of inhibition and longer periods of activity than method 2, and when method 3 was used no activity could be detected. Rubber containing clindamycin hydro- chloride and both the fusidates gave large zones of inhibition, and the antibacterial activity when tested by serial plate transfer persisted for relatively long periods. The periods of persistence of antibacterial activity were less when the elution method was used than when the serial plate transfer test was used (Figs 1 and 2). Because of the long series of daily

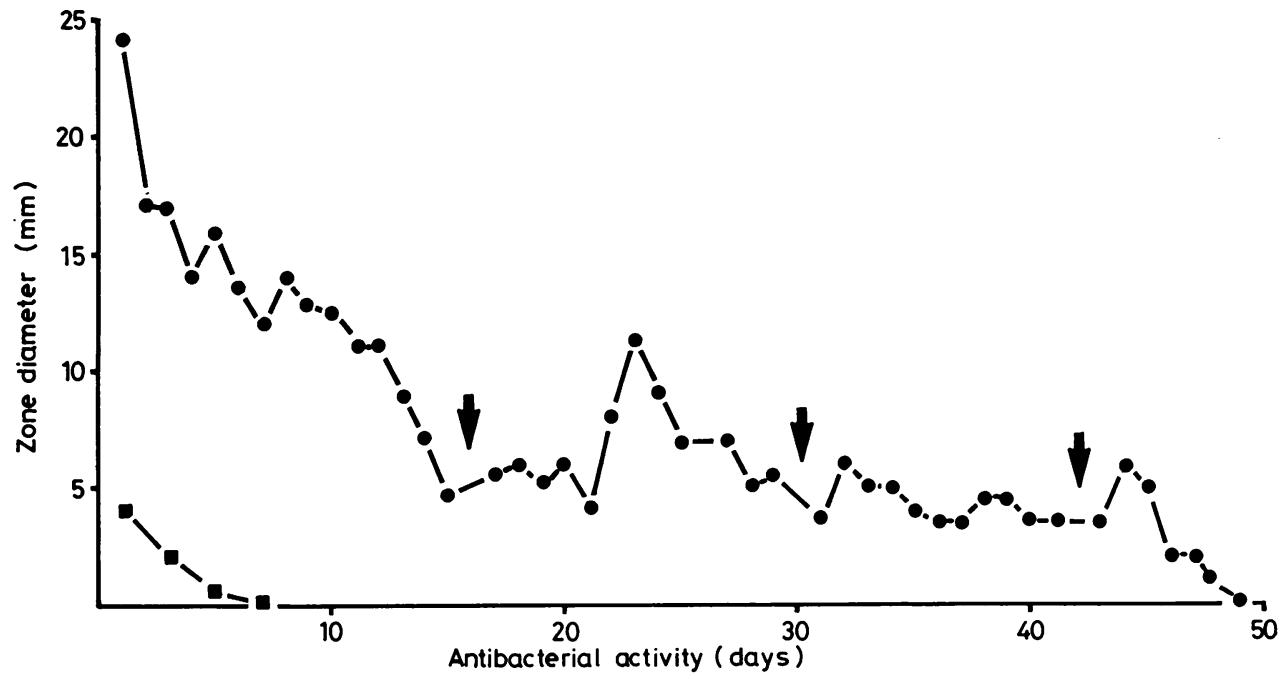

Fig. 1 The antibacterial activity of silicone rubber containing diethanolamine fusidate, introduced by milling the drug into the rubber before processing (method 1). The rubber was tested by the elution method ( $\square$ - and the serial plate transfer method (- - O). Periods of prolonged incubation between tests ure indicated by arrows. Each point represents the mean of three tests.

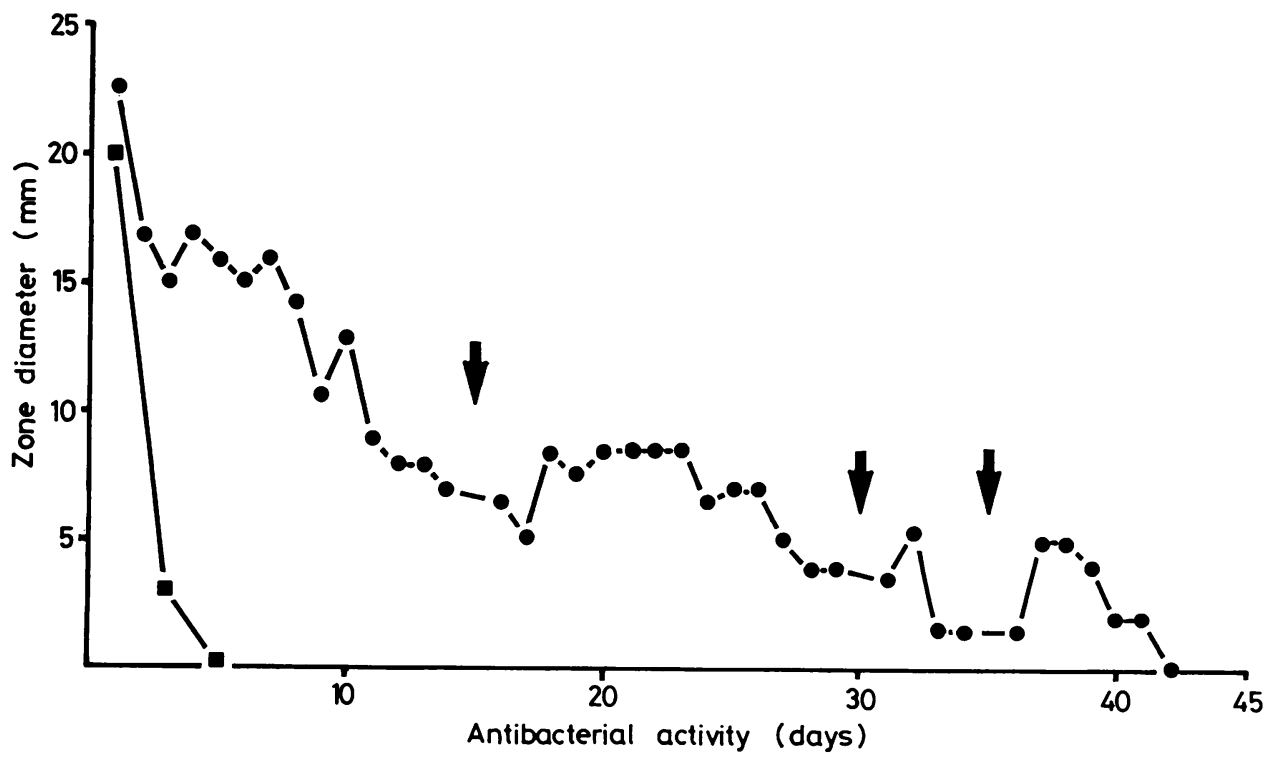

Fig. 2 The antibacterial activity of silicone rubber containing diethanolamine fusidate, introduced by immersion of the processed rubber in a chloroform solution of the drug (method 4). The rubber was tested by the elution method ( $\square$ ) and the serial plute transfer method (O-Periods of prolonged incubation between tests are indicated by arrows. Each point represents the mean of three tests. 
tests, staff were not always available and discs were occasionally left on the test plates for two days instead of the intended one day's incubation. These episodes are indicated in the Figures and were always followed by a peak of renewed activity.

When "depleted" eluted discs were tested by the layered plate method, all except those containing gentamicin sulphate showed increasing zones of inhibition with refrigeration time (Table 2).

Table 2 Zones of activity in mm of "depleted" discs of silicone rubber containing drugs, tested by the layered plate method for slow release

\begin{tabular}{lllll}
\hline Drug & $\begin{array}{l}\text { Method of } \\
\text { introduction }\end{array}$ & $\begin{array}{l}\text { Number of days } \\
\text { refrigerated }\end{array}$ & \\
\cline { 2 - 5 } & & 0 & 3 & 7 \\
\hline Gentamicin sulphate & 1 & 0 & 0 & 0 \\
Sodium fusidate & 1 & 1 & 2 & 4 \\
Diethanolamine fusidate & 1 & 1 & 3 & 4 \\
Diethanolamine fusidate & 4 & 0 & 2 & 5 \\
Clindamycin hydrochloride & 4 & 1 & 3 & 6 \\
\hline
\end{tabular}

\section{Discussion}

The antimicrobial agents studied were chosen initially because of their spectra of activity, particularly against staphylococci, although the use of medical grade silicone rubber in other implants such as cardiac defect patches and valves, small joint replacements and central venous catheters made a wider antimicrobial spectrum desirable. Gentamicin sulphate is active against most enterobacteria such as Escherichia coli, and most strains of Staph aureus. In recent years, a high proportion of "hospital strains" of Staph albus have shown resistance to this antibiotic, but most strains isolated from shunts are still sensitive. Fucidin is almost exclusively antistaphylococcal, and while resistant strains of both Staph aureus and Staph albus occur, they are uncommon. Clindamycin hydrochloride is active against most staphylococci, and against many streptococci and anaerobes. The formaldehyde-urea condensate shave a wide spectrum of activity which includes yeasts and all of the bacteria except some strains of pseudomonas. The antiseptics have the additional advantage in that their use is unlikely to lead to the emergence of resistant strains. Another important criterion was that the agents must have been used clinically without evidence of serious toxicity or allergenicity. Preliminary investigation showed that the rate of release of drugs from silicone rubber was slow, but even if all the drug in an impregnated cerebrospinal fluid (CSF) shunt or central venous catheter were released instantly on implantation the total dose would be too low to cause toxicity. Slow release of extremely small amounts of drug over a long period was also considered to be unlikely to give rise to untoward effects, although recent work has suggested that parenteral, as well as oral, clindamycin may cause pseudomembraneous enterocolitis (DW Burdon, personal communication, 1981). None of the agents tested was considered to be allergenic.

The formaldehyde-urea condensates gave no detectable activity after introduction into the rubber using method 1 . It is unlikely that the drugs decomposed during processing, as another important criterion for the choice of drugs for this study was heat stability. The drugs had to withstand the temperatures of crosslinking and routine autoclaving, as any devices made from impregnated rubbers would need to be sterilised before use. Their lack of activity, and the differences in results between the other drugs and between the two different test methods, may be explained by their physicochemical properties in relation to those of silicone rubber.

Studies on the theory of release of agents from matrices ${ }^{10-12}$ indicate that several factors are involved. The important ones in the case of heat-crosslinked silicone rubber matrix are the molecular weight of the drug, and its solubility in the matrix. The diffusibility of the drug in the matrix falls with increase i molecular weight, and while the exact moleculat weights of formaldehyde-urea condensates are nơ known due to the variable chain length of the polymers, they are probably at least an order of magnitude higher than those of other drugs such as gentamicin sulphate (543), diethanolamine fusidate (605), sodium fusidate (538) and clindamycin hydrochloride (425). The solubility $(\mathrm{Cm})$ of a drug in a crosslinked matrix is difficult to determine experimentally and is usually inferred indirectly. The solubility of the drug in a solvent in which the matrix, or its uncrosslinked form, is highly soluble is usually accepted as an indirect indication of $\mathrm{Cm} .{ }^{11}$ Uncrosslinked silicone rubber is soluble in chloroform, and the solubility of drugs in this solvent is easily determined. Both of the formaldehyde-urea condensates are insoluble in chloroform, and this is an additional factor which may explain their lack of $\frac{D}{O}$ activity. However, gentamicin sulphate is also insoluble in chloroform, and when incorporated in $N$ silicone rubber it conferred activity which persisted for a short time when the drug powder was milled directly into the rubber, or when the first emulsion method was used. The second emulsion method (method 3) resulted in loss of activity. The difference $\mathscr{C}$ between the two gentamicin sulphate-containing $\stackrel{\Phi}{\Phi}$ rubbers prepared by the emulsion methods is in the ? drug particle size. Gentamicin sulphate in rubber prepared by method 2 has a particle diameter 
ranging from more than $25 \mu \mathrm{m}$ to less than $0.5 \mu \mathrm{m}$, whereas in that resulting from method 3 most of the particles are less than $0.5 \mu \mathrm{m}$ in diameter. ${ }^{9}$ Due to the insolubility of gentamicin sulphate in the matrix, and its high water solubility, any antibacterial activity of rubber containing this drug is likely to be due only to dissolution of drug particles which lie at the surface of the rubber and are incompletely incarcerated, and not to diffusion through the matrix. The smaller drug particle size and more efficient distribution in the matrix results in less drug being available at the surface for dissolution. Also, the smaller particles are more likely to be completely incarcerated.

Clindamycin hydrochloride and diethanolamine fusidate are soluble in both chloroform and water and while initial antibacterial activity may be due to dissolution of particles directly accessible from the rubber surface, the greater degree of persisting activity is probably due to their ability to diffuse through the matrix.

The peaks of activity shown in Figs 1 and 2 when the release of diethanolamine fusidate was studied by the serial plate transfer method all followed periods of prolonged incubation. An unbroken series of daily tests over six weeks, carried out in a laboratory with two members of staff, proved impossible to maintain. Occasions arose when tests had to be postponed for a day, resulting in discs remaining in contact with the same plate for two days. These occasions were always followed by an increase in the size of the inhibition zones, suggesting that diffusion through the rubber had been slowed by the increase of drug in the agar surrounding the discs, resulting in an accumulation of drug which was released when the discs were transferred to a fresh plate.

Two methods of testing were used in order to investigate the effects of different environments. The elution test simulates fluid environments such as the inside of a CSF shunt or central venous catheter. The serial plate transfer method simulates an aqueous non-fluid environment such as connective tissue. The exact conditions in which such implants are used are impossible to simulate in vitro, and they may vary considerably for different parts of the same implant, one end of which may be immersed in CSF and the other end in blood, with the intervening portions passing through brain, bone, fat, muscle and connective tissue. However, the results of the two tests demonstrate that drug release under these conditions may vary considerably. The concentrations of drug in the elution fluid and agar are probably very low, but the aim is not to release therapeutically active amounts of drug into the surrounding tissues, but to give sufficient concentrations of drug at the surface of the rubber to prevent colonisation from occurring. In the case of CSF shunts, colonisation has been shown to be due to the development of microcolonies on the luminal surfaces of the tubes, rather than to organisms suspended in the CSF. ${ }^{13}$

Release in the serial plate transfer method was slower than in the elution method, where the drug which leaves the rubber was immediately diluted throughout the eluting fluid and not allowed to accumulate. This rapid rate of removal of the drug from the periphery of the rubber probably exceeded the maximum diffusion rate of the drug through the rubber, resulting in temporary depletion at the periphery and negative results on testing. The results of the layered plate test for slow release show that this situation was temporary (Table 2). These results also indicate that loss of activity in rubber containing gentamicin sulphate is not due to this phenomenon.

The period of risk of shunt colonisation is during the operation to insert or revise the shunt, and haematogenous infection has not been shown to occur afterwards. ${ }^{14-16}$ In order to cover this risk, a short period of antibacterial activity would suffice, but in order to achieve this using gentamicin sulphate the majority of the drug would have to remain fixed in the matrix. Also, revision of the system soon after insertion is not uncommon, presenting a further risk of colonisation. The use of a longer acting preparation such as diethanolamine fusidate would cover revisions required in the month following insertion, and it may also allow shunts to be inserted in patients who have pre-existing sepsis, with less risk of consequent colonisation of the shunt. The possibility of using method 4 to impregnate commercially available shunt catheters removes the difficulties inherent in the commercial use of the other methods.

We have found no significant effect on mechanical properties of impregnating silicone rubber $^{17}$ and studies of more subtle effects on slit valve function using Dow Corning valves have so far been satisfactory. ${ }^{18}$ Further studies, including thrombogenicity tests, must be undertaken before impregnated catheters are used clinically.

We are grateful to Miss Julie Swinden and Mrs Rose Davies for technical assistance, and Mrs Joan Billington for typing the manuscript. The work was supported by a grant from the Association for Research into Spina Bifida and Hydrocephalus, and was conducted during the tenure of the ASBAH Research Fellowship by RB.

Antimicrobial drugs were kindly donated free by the manufacturers.

\section{References}

${ }^{1}$ Bayston R, Lari J. A study of the sources of infection in 
colonised shunts. Dev Med Child Neurol [Suppl] 1974;32: 16-22.

2 Anderson FM. Ventriculo-auriculostomy in treatment of hydrocephalus. J Neurosurg 1959;16:551.

${ }^{3}$ Tsingoglou S, Forrest DM. Complications from Holter ventriculo-atrial shunts. BrJ Surg 1971;58:372-7.

4 James HE, Walsh JW, Wilson HD, Connor JD, Bean JR, Tibbs PA. Prospective randomised study of therapy in cerobrospinal fluid shunt infection. Neurosurgery 1980; 7, 5:459-63.

${ }^{5}$ Bayston R. Antibiotic prophylaxis in shunt surgery. Dev Med Child Neurol [Suppl] 1975;35:99-103.

${ }^{6}$ Welch K. The prevention of shunt infection. Zeit für Kinderchirurg 1977;22, 4:265.

7 Welch, K. Residual shunt infection in a programme aimed at its prevention. Zeit für Kinderchirurg 1979;28, 4:374-7.

${ }^{8}$ Bayston R. The antibacterial effects of impregnated silastic and its possible applications in surgery. $J$ Pediatr Surg 1977;12, 1:55-61.

${ }^{9}$ Bayston R. The impregnation of medical silicone elastomers and acrylic bone cements with antimicrobial drugs. Ph D Thesis, University of Sheffield, 1979.

${ }^{10}$ Higuchi T. Rate of release of medicaments from ointment bases containing drugs in suspension. J Pharm Sci 1961; 50: 874-5.

${ }^{11}$ Baker RW, Lonsdale HK. Controlled release-mechanisms and rates. In: Controlled release of biologically active agents. Tanquary and Lacey, Plenum Publications, 1974.
12 Flynn GL. Influence of physicochemical properties of drug and system on release of drugs from inert matrices. In: Controlled release of biologically active agents. Tanquary and Lacey, Plenum Publications, 1974.

${ }^{13}$ Bayston R, Penny SR. Excessive production of mucoid substance in Staphylococcus SIIA. A possible factor in colonisation of Holter shunts. Dev Med Child Neurol [Suppl] 1972; 14, 27: 25-8.

${ }^{14}$ Bayston R. Serological surveillance of children with CSF shunting devices. Dev Med Child Neurol [Suppl] 1975: 17, 35:104-10.

${ }^{15}$ Bayston R, Spitz L. The role of retrograde movement of bacteria in ventriculo-atrial shunt colonisation. Zeit für Kinderchirurg 1978;25, 4:352-6.

${ }^{16}$ Bayston R, Swinden J. The aetiology and prevention of shunt nephritis. Zeit für Kinderchirurg 1979;28, 4:377-84.

17 van Noort R, Bayston R. Mechanical properties of antibacterial silicone rubber for hydrocephalus shunts. J Biomed Mater Res 1979;13:623-30.

${ }^{18}$ Bayston R. The effect of antibiotic impregnation on the function of slit valves used to control hydrocephalus. Zeit für Kinderchirurg 1980;31, 4:353-9.

Requests for reprints to: Dr $\mathrm{R}$ Bayston, Lecturer in Bacteriology, Department of Paediatric Surgery, Institute of Child Health, 30 Guilford Street, London WC1N $1 \mathrm{EH}$, England. 